\title{
Customized Requirements Driven Multivariate Quality Control for Steelmaking Process
}

\author{
Jingping Yang ${ }^{1, a}$, Wanlei Wang ${ }^{1, b}$ Jia $\mathrm{Xu}^{1, \mathrm{c}}$, and Shoufang $\mathrm{Mi}^{1, \mathrm{~d}}$ \\ ${ }^{1}$ Dalian University of Nationalities, Liaoning province, China

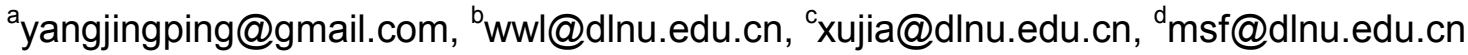

Keywords: Customer Requirements; Process quality control; Principal component analysis (PCA); Multivariate control chart

\begin{abstract}
In this paper, a new SPC based quality control process model for steelmaking industry is established, in which a Customer Requirements Weighted-Principal Component Analysis (CRW-PCA) method is proposed, the multivariate control charts based on this method can make special emphasis on the controlling of steelmaking quality characters response to customer's special requirements. Practices show that compared with the traditional PCA-based multivariate control chart, the multivariate control charts based on CRW-PCA is more adaptive to the needs of today's process quality control of steelmaking due to the adequate consideration of customers' requirements.
\end{abstract}

\section{Introduction}

In recent years, the competitive environment the steelmaking enterprises are facing changed greatly, the customer's requirements on the variety, specification and performance of the steel products tends to be more and more diverse, while the demanding to products quality is also increasing. Due to the diversity customer requirements, complex process flow, multiple production constraints and its special product hieratical construction, the production process of the steelmaking industry presents highly complicated dynamic characteristics. ${ }^{[1]}$ Since multi-variables should be monitored and controlled in steelmaking quality control, which can be seen a multi-variable quality control ${ }^{[2]}$ problem. Primary Content Analysis (PCA) is one of a relatively mature data compression and indices dimension reduction technology. ${ }^{[3]}$ However, traditional PCA treat every ingredient equally, so the customer requirements' specialties are often not considered which also leads to over control or under control of the production process.

In order to work out these problems, a Customized Requirements Weighted- Principal Component Analysis (CRW-PCA) is proposed in this paper. The method can make up the traditional PCA's deficiency of compressing data on average, give full consideration to the special needs of customers, and Help enterprises to practice customers centered principle of quality management.

\section{Process quality control model for steelmaking industry}

Current widespread implementation and practice of statistical process control (SPC) attest to the potential of SPC to contribute to continuous quality improvement efforts. ${ }^{[4]} \mathrm{A}$ primary tool used for SPC is the control chart, a graphical representation of certain descriptive statistics for specific quantitative measurements of the manufacturing process. These descriptive statistics are displayed in the control chart in comparison to their "in-control" sampling distributions. The comparison detects any unusual variation in the manufacturing process, which could indicate a problem with the process.

Steelmaking is a continuous, uninterrupted process from high-temperature melting of all kinds of raw materials to ingots or even slabs to be formed, all the materials are mixture of fluid. Hence, chemical elements' content turns to be some of the main control objects as critical process quality characteristics. 
While quality control for steel industry is a serial of process technical activities that aims at monitoring processes and removing failures and unsatisfied factors during all the quality phases to achieve quality requirements and economic benefits. ${ }^{[5]}$

A quality control model based on SPC theories as shown in Fig. 1 is proposed in this paper. The quality control process is goes in a parallel and synergetic way with process design and manufacturing, which both acts as the results and sponsor to the quality control, hence the whole quality control process is to appear as a closed loop. The main process of the SPC based quality control mainly includes five critical processes: 1) critical characteristics recognition; 2) data collection and preconditioning. 3) control chart generation; 4) process capacity estimation; and if the control chart is stable 5) standardizing the results into the enterprise standard database; or if the control chart is not stable 6) improve the related processes.

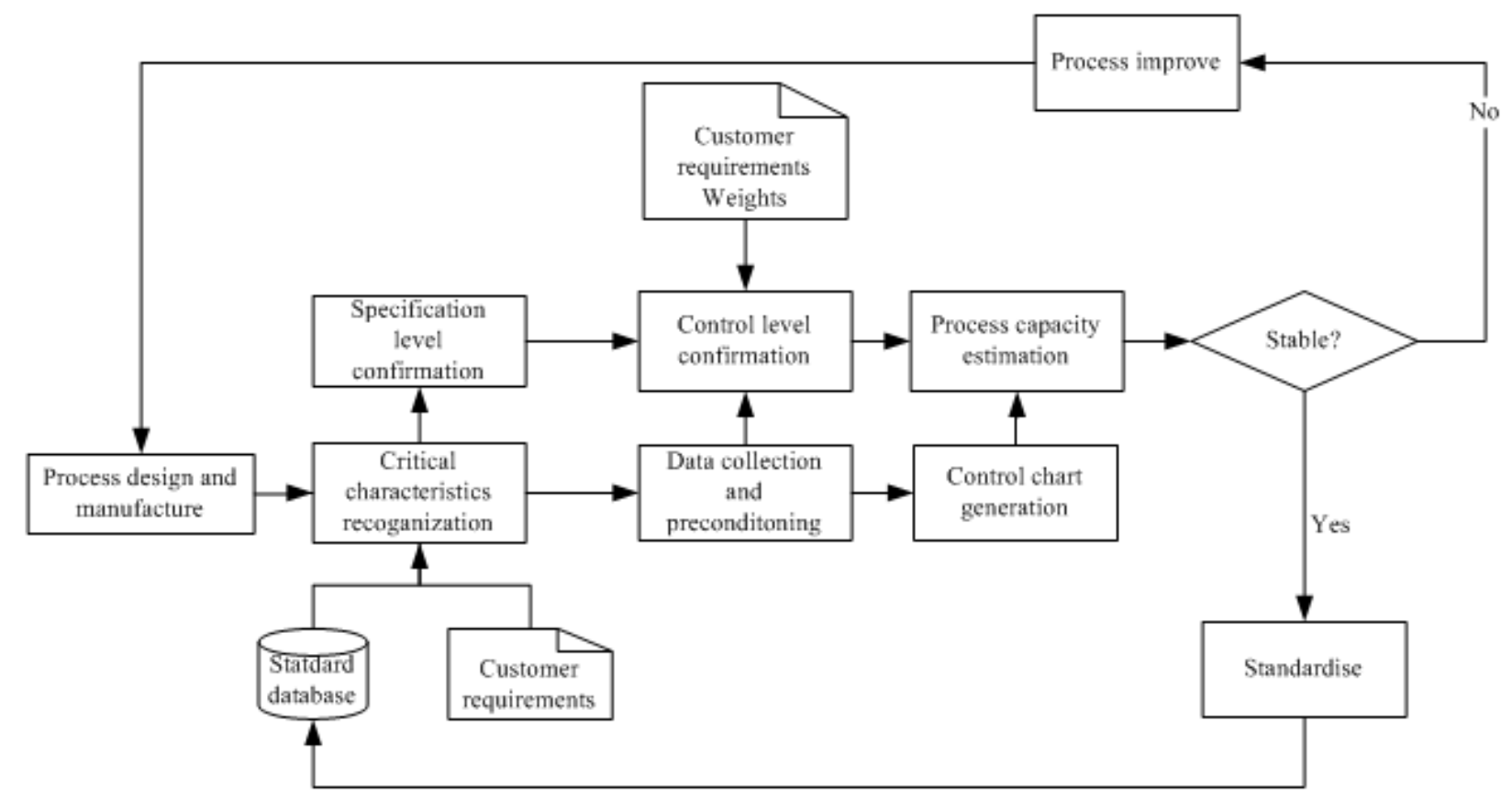

Fig.1 SPC based quality control process model for steelmaking industry

\section{Critical quality characteristics recognition and data precondition}

According to the process design requirements and the standard database, we can recognize all the traditional quality control characteristics, but customers often have some special requirements and mostly are quite more strict than the relative standards. So when we discover the critical characteristics, how to dig the customers requirements which are often inconspicuous, not quite clear and nonstandard.

A mature resolution for this problem is QFD or fuzzy QFD method which can easily turns customers obscure requirements into critical quality characteristics as shown in Fig.2. 


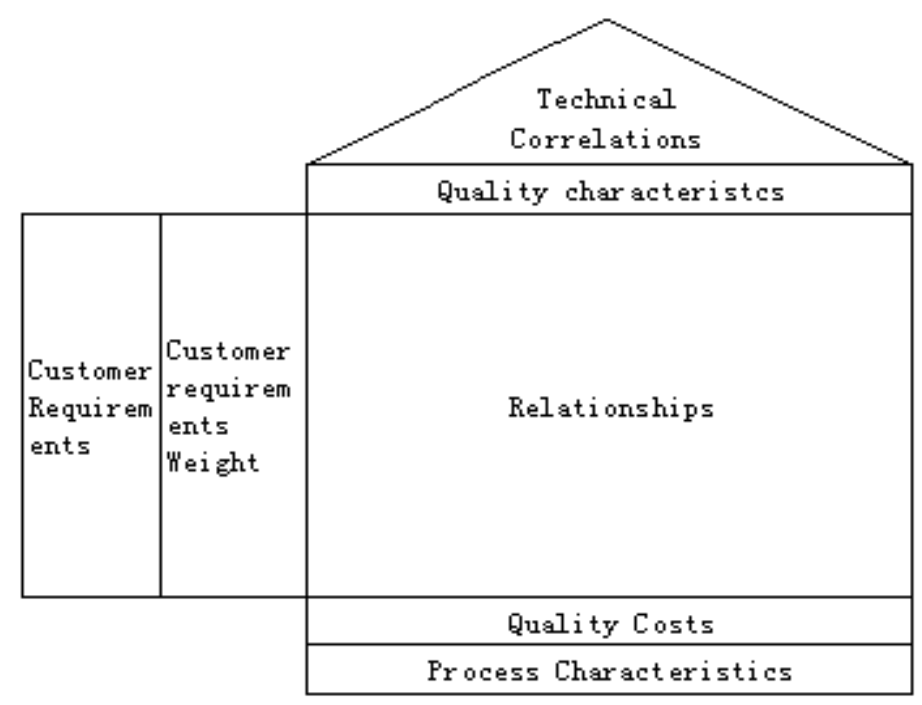

Fig. 2 HoQ for critical characteristics recognition

The critical quality characteristics are variable along with the different customers. In this paper, we choose the smelting inspection data of 10 elements $(\mathrm{C}, \mathrm{Mn}, \mathrm{Si}, \mathrm{P}, \mathrm{S}$, etc) of the product G20CrNi2MoA under the standard YB4100-1998 as the quality characteristics to present the procedure of statistical analysis of process quality characteristics. We randomly extract 60 records from the inline quality inspection data as shown in Table 1. For $n$ groups of $p$ characteristics' test results data $X=\left(x_{i j}\right)_{n \times p}$, although they are dimensional consistency(\%), they have an order of magnitude difference, so we have to diminish these difference by another magnitude standardization to access $Y=\left(y_{i j}\right)_{n \times p}$, where $y_{i j}=\left(x_{i j}-\bar{x}_{j}\right) / s_{j}$ is each standardization value of $x_{i j}, \bar{x}_{j}$ and $s_{j}$ are the average and the standard variation of the elements in sample $j$, respectively. As in this example, the standardized processing results are shown in Table 2.

Table 1 Raw data of elements (\%)

\begin{tabular}{ccccccc}
\hline $\mathrm{n}$ & $\mathrm{C}$ & $\ldots$ & $\mathrm{P}$ & $\mathrm{S}$ & $\ldots$ & $\mathrm{Ti}$ \\
\hline 1 & 0.200 & $\ldots$ & 0.011 & 0.002 & $\ldots$ & 0.0010 \\
2 & 0.180 & $\ldots$ & 0.007 & 0.003 & $\ldots$ & 0.0007 \\
3 & 0.200 & $\ldots$ & 0.007 & 0.003 & $\ldots$ & 0.0010 \\
& & & $\ldots$ & & & \\
60 & 0.190 & $\ldots$ & 0.012 & 0.007 & $\ldots$ & 0.0030 \\
\hline
\end{tabular}

Table 2 Standardized data of elements $(\%)$

\begin{tabular}{ccccccc}
\hline $\mathrm{n}$ & $\mathrm{YC}$ & $\ldots$ & $\mathrm{YP}$ & $\mathrm{YS}$ & $\ldots$ & $\mathrm{YTi}$ \\
\hline 1 & 0.0836 & $\ldots$ & 0.1121 & -1.1551 & $\ldots$ & -0.6500 \\
2 & -1.9235 & $\ldots$ & -1.5697 & -0.7700 & $\ldots$ & -1.0374 \\
3 & 0.0836 & $\ldots$ & -1.5697 & -0.7700 & $\ldots$ & -0.6500 \\
& & & $\ldots$ & & & \\
60 & -0.9199 & $\ldots$ & 0.5326 & 0.7700 & $\ldots$ & 1.9328 \\
\hline
\end{tabular}

\section{Customer requirement weighted process}

Suppose there are $\mathrm{p}$ critical quality characteristics need to be monitored and controlled, and the strict level of these characteristics can be standardized to a weight vector $W=\left(w_{1}, w_{2}, \cdots, w_{p}\right)$, where $0<w_{j}<1$ and $\sum_{j=1}^{p} w_{j}=1$.In this paper, we weighted the standardized processing results to get a matrix $Z=\left(z_{i j}\right)_{n \times p}=\left(w_{j} y_{i j}\right)_{n \times p}$. 
In this example, the customer has special requirements on characteristics $\mathrm{C}, \mathrm{P}$ and $\mathrm{S}$ as $\mathrm{C} \leq 0.22$, $\mathrm{P} \leq 0.015$ and $\mathrm{S} \leq 0.007$. According to the distances to the relative standard regulation of $\mathrm{C} \leq 0.23$, $\mathrm{P} \leq 0.02, \mathrm{~S} \leq 0.02$, we determine the customer requirement level for these three characteristics are: low, middle and high. For the other characteristics, we determine they should be specified by standard YB4100-1998. So the strict level of the customer requirements can be present by a vector $\mathrm{W}=(0.1$, $0.05,0.05,0.15,0.25,0.05,0.05,0.05,0.05,0.05)$, where the weight of characteristics element $\mathrm{C}, \mathrm{P}$ and $\mathrm{S}$ are $0.1,0.15$ and 0.25 , respectively, and other weight are 0.05 equally. After the customer requirement-weighted process the standardized data of elements is turned to be $\mathrm{ZC}, \mathrm{ZP}$ and $\mathrm{ZS}$ etc, as shown in Table. 3.

Table 3 Customer requirement-weighted data of elements (\%)

\begin{tabular}{ccccccc}
\hline $\mathrm{n}$ & $\mathrm{ZC}$ & $\ldots$ & $\mathrm{ZP}$ & $\mathrm{ZS}$ & $\ldots$ & $\mathrm{ZTi}$ \\
\hline 1 & 0.0084 & $\ldots$ & 0.0168 & -0.2888 & $\ldots$ & -0.0325 \\
2 & -0.1923 & $\ldots$ & -0.2355 & -0.1925 & $\ldots$ & -0.0519 \\
3 & 0.0084 & $\ldots$ & -0.2355 & -0.1925 & $\ldots$ & -0.0325 \\
& & & $\ldots$ & & & \\
60 & -0.0920 & $\ldots$ & 0.0799 & 0.1925 & $\ldots$ & 0.0966 \\
\hline
\end{tabular}

\section{Primary Content Analysis method for CRW data}

After weighted processing, the characteristics mostly exist positive or negative correlation, For this example, the correlation between vectors of elements are shown in Table.4, so we have to compress the data by PCA method.

Table 4 Correlation between vectors of elements

\begin{tabular}{|c|c|c|c|c|c|c|}
\hline & $\mathrm{C}$ & $\ldots$ & $\mathrm{P}$ & $\mathrm{S}$ & $\ldots$ & $\mathrm{Ti}$ \\
\hline $\mathrm{C}$ & 1.000 & $\ldots$ & -0.117 & -0.013 & $\ldots$ & -0.147 \\
\hline $\mathrm{P}$ & -0.117 & $\ldots$ & 1.000 & 0.189 & $\ldots$ & 0.326 \\
\hline $\mathrm{S}$ & 0.122 & $\ldots$ & 0.189 & 1.000 & $\ldots$ & 0.549 \\
\hline $\mathrm{Ti}$ & -0.147 & $\ldots$ & 0.326 & 0.549 & $\ldots$ & 1.000 \\
\hline
\end{tabular}

Firstly, a relationship matrix $C_{p \times p}=\operatorname{correcoef}(Z)$ should be worked out by matlab relationship function, and then the Eigenvalue and Eigenvector of matrix $C$ are solved by the Jacobi Method. The principal component extraction results is shown in Table 5.

Table 5 The principal component extraction

\begin{tabular}{cccc}
\hline & \multicolumn{3}{c}{ Original eigenvalues } \\
\cline { 2 - 4 } & Eigenvalues & Contribution of variance & Cumulative contribution \\
& & $(\%)$ & $(\%)$ \\
2 & 2.570 & 25.699 & 25.699 \\
3 & 1.832 & 18.323 & 44.021 \\
4 & 1.178 & 11.784 & 55.805 \\
& 1.087 & 10.875 & 66.680 \\
9 & & $\ldots$ & 97.711 \\
10 & 0.324 & 3.239 & 100.000 \\
\hline
\end{tabular}


According to the cumulative variance contribution rate corresponding to each Eigenvalue in Table 5 , we can determine the number of principal components $\mathrm{m}(4$ for this example), and the load coefficient of these 4 components are up to $66.68 \%$.

The square root of the load coefficient of each component divided by corresponding Eigenvalue is obtained which make the corresponding coefficient of the principal component expressions, the expressions is like below:

$$
\begin{aligned}
& F_{1}=a_{11} Z_{1}+a_{12} Z_{2}+\cdots+a_{1 p} Z_{p} \\
& F_{2}=a_{21} Z_{1}+a_{22} Z_{2}+\cdots+a_{2 p} Z_{p} \\
& F_{m}=a_{m 1} Z_{1}+a_{m 2} Z_{2}+\cdots+a_{m p} Z_{p}
\end{aligned}
$$

where $a_{i 1}, a_{i 2}, \cdots, a_{i p}(i=1,2, \cdots, m)$ is the Eigenvector corresponding to the Eigenvalue of the matrix $\sum$ which is the covariance matrix of $Z$, and $Z_{1}, Z_{2}, \cdots, Z_{p}$ is the weighted and standardized value of the original data, as in this example the functions are like below:

$$
\begin{aligned}
& F_{1}=0.233 Z C+0.331 Z M n+0.372 Z S i-0.271 Z P-0.427 Z S+0.142 Z C r \\
& +0.369 Z M o+0.363 Z N i-0.092 Z C u-0.382 Z T i \\
& F_{2}=0.048 Z C+0.406 Z M n+0.103 Z S i+0.213 Z P+0.264 Z S+0.532 Z C r \\
& +0.081 Z M o+0.124 Z N i+0.564 Z C u+0.293 Z T i \\
& F_{3}=0.452 Z C+0.300 Z M n+0.452 Z S i-0.086 Z P+0.291 Z S-0.135 Z C r \\
& -0.126 Z M o-0.313 Z N i-0.402 Z C u+0.338 Z T i \\
& F_{4}=-0.162 Z C-0.033 Z M n+0.399 Z S i+0.650 Z P-0.275 Z S-0.437 Z C r \\
& +0.158 Z M o+0.178 Z N i+0.071 Z C u+0.248 Z T i
\end{aligned}
$$

A comprehensive value of each principal component should be worked out by equation (1.1) (1.4) as shown in Table.6, and then we can draw a multivariate control chart of average $\bar{x}$ and range $R$.

Table 6 Scores of original data and synthesis value

\begin{tabular}{llllll}
\hline $\mathrm{n}$ & $\mathrm{F} 1$ & $\mathrm{~F} 2$ & $\mathrm{~F} 3$ & $\mathrm{~F} 4$ & Synthesis value \\
\hline 1 & 0.1380 & -0.1647 & -0.0791 & 0.1237 & 0.0180 \\
2 & 0.1060 & -0.1340 & -0.1088 & 0.0028 & -0.1340 \\
3 & 0.3144 & 0.0135 & -0.0428 & -0.1002 & 0.1850 \\
4 & 0.1480 & -0.0910 & -0.0876 & -0.0176 & -0.0482 \\
$\ldots \ldots$ & & & & & \\
59 & -0.1112 & 0.1224 & 0.0089 & -0.0176 & 0.0026 \\
60 & -0.0967 & 0.2407 & 0.1105 & 0.0659 & 0.3203 \\
\hline
\end{tabular}

\section{Multivariate control chart and comparison analysis}

In order to observe the results, we divided 60 comprehensive values from tab. 6 into 30 samples, each sample is consists of two data. Put these data into SPSS or matlab software, we can obtain the multivariate control charts of average $\bar{x}$ and range $R$ as shown in Fig.3 and Fig.4. 


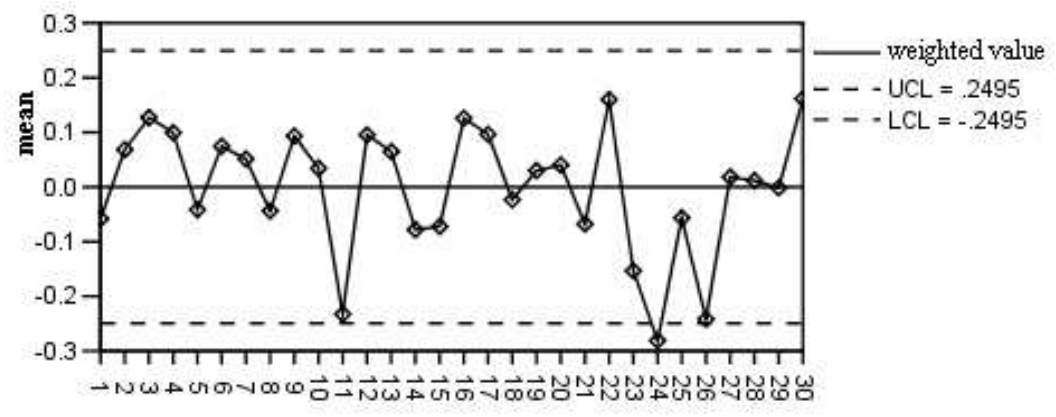

Fig. 3 CRW-PCA-based multivariate $\bar{x}$-control chart

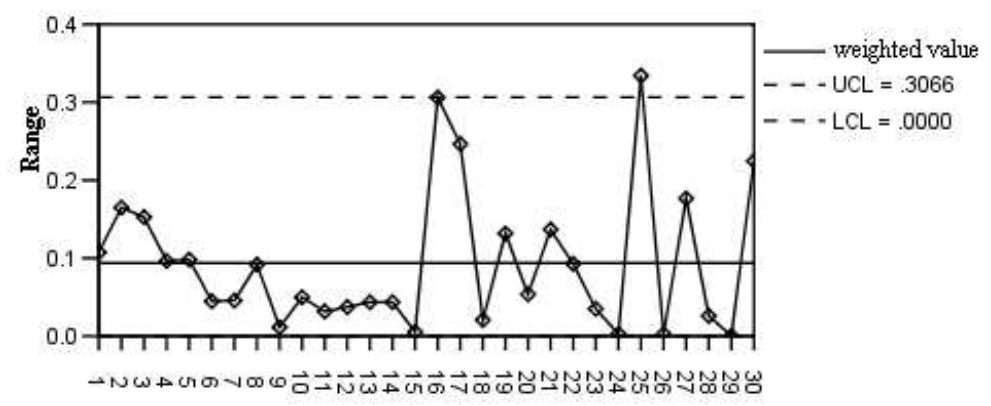

Fig.4 CRW-PCA-based multivariate $R$-control chart

In practice, we can judge whether the steel components are in control intuitively and quickly. These judge conditions include: 1) Whether all of the points are inside the control line; and 2) Whether all of the points are presenting as a random distribution.

In order to show the differences between the CPW-PCA based multivariate control chart and the traditional PCA based multivariate control chart, we also draw the traditional PCA based multivariate control charts as shown in Fig.5 and Fig. 6 with raw data in Table1. Obviously, according to traditional PCA multivariate control charts, all of the comprehensive value are in control as shown in Fig.5 and Fig.6, but the CRW-PCA multivariate control charts show that some comprehensive values are on or out of the control line. So although the process is stable, but there are some tiny distances from the customer special requirements, the process needs to be improved yet.

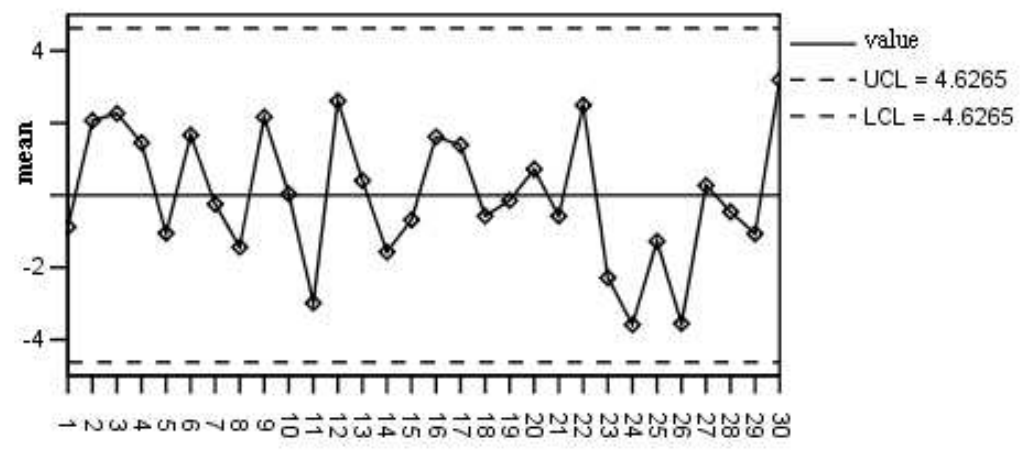

Fig.5 Traditional PCA-based multivariate $\bar{x}$-control chart 


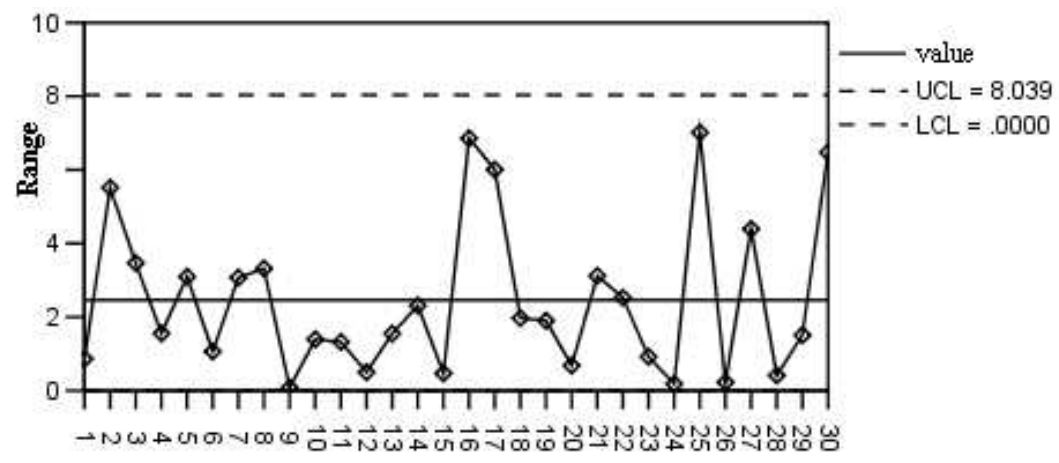

Fig.6 Traditional PCA-based multivariate $R$-control chart

\section{Summary}

In conclusion, the CRW-PCA based multivariate control chart is helpful in adjusting the control requirements response for the customer's special requirement, hence it is able to diminish possible under-control or over-control problem of the process, and has some advantages to the traditional PCA based multivariate quality control method.

Supported by "the Fundamental Research Funds for the Central Universities"

\section{References}

[1] Xiaobing Liu, Caiyan Liu, Yue Ma, Guang Yang. Attribute model based integrated quality management technologies for steel and iron industries. Manufacturing Automation. 26(2004) $42-46$.

[2] C.-Y. Chou, H.-R. Liu, X.R. Huang, etal. Economic-Statistical Design of Multivariate Control Charts Using Quality Loss Function.The International Journal of Advanced Manufacturing Technology. 20(2002) 916-924.

[3] Jinghua Yu, Weiquan Yang. Multivariate statistical analysis and Application. Zhongshan University press, Guangzhou, 2005, 2.

[4] Manus Rungtusanatham, John C. Anderson and Kevin J. Dooley. Conceptualizing Organizational Implementation and Practice of Statistical Process Control. JOURNAL OF QUALITY MANAGEMENT, 2 (1997).

[5] Zhuo Zhang. Quality control for steel products analysis. Metallurgical analysis, 6 (2005) 82-84. 